USITP-94-14

October 1994

\title{
The heavy quark potential in two-dimensional QCD with adjoint matter
}

\author{
T.H. HANSSON ${ }^{\dagger}$ AND R. TZANI ${ }^{\dagger}$ \\ Stockholm University, Department of Physics, \\ Box 6730, S-11385 Stockholm, SWEDEN
}

\begin{abstract}
Using a loop formulation approach of $\mathrm{QCD}_{2}$, we study the potential between two heavy quarks in the presence of adjoint scalar fields, and demonstrate how 't Hooft's planar rule is manifested in this formulation. Based on some physical assumptions, we argue that large adjoint loops "confined" inside an external fundamental one give a Casimir type contribution to the potential energy, while the small loops only renormalize the string tension. We also extend the results to the case of massive adjoint fields.
\end{abstract}

$\dagger$ hansson@vana.physto.se; supported by the Swedish Natural Sience Research Council

$\ddagger$ tzani@vana.physto.se; address after September 1994: Imperial College, Physics Dept., Prince Consort Road, London SW72BZ 


\section{Introduction}

Ever since the original work by 't Hooft [1], two-dimensional QCD $\left(\mathrm{QCD}_{2}\right)$, has been an important "laboratory" for testing various ideas about strong interaction physics. Early work established a string-like meson spectrum and a parton picture for $e^{+} e^{-}$-production and deep inelastic scattering. The finite temperature behaviour was studied [2], and the connection to string theory was worked out in some detail in a series of papers by Bars and Hansson [3], who showed that to leading order in $1 / N$ 't Hooft's result follows from a string model with quarks attached to the ends. Later, Strominger derived this string Lagrangian directly from QCD by considering expectation values of Wilson loops and using factorization at large $N[4]$.

In a related development Makeenko and Migdal [5] reformulated Yang-Mills theory in loop variables and Kazakov and Kostov [6] managed to solve the loop equations, for the case of $\mathrm{QCD}_{2}$, and obtained closed equations for the expectation values of arbitrary Wilson loops in the limit $N \rightarrow \infty$. This approach was extended to finite $N$ by Kazakov [7].

Recently, there has again been a lot of interest in the connection between QCD and string theory. In particular, by counting the degrees of freedom (d.o.f.) in a QCD string, Polchinski has shown that a string theoretical description of the large $N$ gauge theory would involve an infinite number of d.o.f. at short distances [8]. Another line of research has focused on pure two-dimensional Yang-Mills theory $\left(\mathrm{YM}_{2}\right)$. This theory does not have any field theoretical d.o.f., just as strings in two dimensions do not have any transverse d.o.f.. On a compact space-time, however, the Wilson loops provide quantum mechanical d.o.f., and it turns out that the corresponding partition function, which can be calculated exactly, has a string interpretation [9-13].

Yet another important development concerns two-dimensional Yang-Mills the-

ory coupled to scalar or fermionic adjoint matter $\left(\mathrm{AdQCD}_{2}\right)$. $\mathrm{AdQCD}_{2}$ is a very interesting theory from several points of view. First, in the large $N$ limit, the 
planar adjoint loops are not suppressed, so the planar approximation corresponds to a bona fide sum of fishnet diagrams. ${ }^{\star}$ To this day, there is no known method to perform such sums analytically.

The second reason for studying (scalar) $\mathrm{AdQCD}_{2}$ is that it is the high temperature limit of pure $\mathrm{YM}_{3}$, where the "time"-component of the original gauge field becomes an adjoint scalar in the dimensionally reduced theory. As opposed to the dimensional reduction from $\mathrm{QCD}_{4}$ to scalar $\mathrm{AdQCD}_{3}$, this reduction is not plagued by uncontrollable infrared effects due to magnetic mass generation, but gives an exact description of the static observables in the $T \rightarrow \infty$ limit [15].

In spite of not having any exact solution, even in the large $N$ limit, several things are known about $\mathrm{AdQCD}_{2}$ :

i) In a series of papers, Dalley and Klebanov [16], and Bhnot, Demeterfi and Klebanov [17] have numerically studied $\mathrm{AdQCD}_{2}$ in the large $N$ limit, using the light-cone quantization technique of Brodsky and Pauli. They obtained a discrete spectrum of bound states with a level density vgrowing exponentially with the mass.

ii) Kutasov [18], studied string properties of the theory using the ideas of Polchinski [8] referred to above. He concluded that the theory exhibits a deconfining phase analogous to Hagedorn transition in string theory and that the spectrum consists of an infinite number of Regge trajectories confirming, thus, its "stringy" nature.

The purpose of the present paper is to study $\mathrm{AdQCD}_{2}$ using a "first quantized" formalism, where the partition function is a sum over loops describing the paths of the adjoint particles. In this picture the gauge interaction amounts to multiply each term in the sum with the expectation value of the corresponding Wilson loops (in the adjoint representation). The reason for taking this unconventional approach

\footnotetext{
* This should be contrasted with $\mathrm{QCD}_{2}$ where, in a linear gauge, the planar diagrams include only dressed ladders that can be expressed by Dyson-Schwinger and Bethe-Salpeter type equations with explicitly known kernels.

$\dagger$ For an interesting numerical approach in the content of a purely scalar theory see [14].
} 
to an interacting field theory, is that we will try to exploit the recently developed technology for computing expectation values of Wilson loops. In fact, [19], [6], [7] and [20] give methods for calculating an arbitrary product of Wilson loops in an arbitrary representation. The question is whether this can be put to practical use in the study of $\mathrm{AdQCD}_{2}$.

Since our approach limits us to the study of Wilson loops, the natural quantity to examine is the potential between heavy particles. In particular, we shall study the heavy quark potential, both for finite and infinite $N$. In the large $N$ limit, we know that the string tension of $\mathrm{QCD}_{2}$ with fundamental quarks is not renormalized by quark loops (since they are down by factors $1 / N$ ), while for $\mathrm{AdQCD}_{2}$ this is not the case. In the "first quantized" version of the theory, even this basic result is not obvious, but, as shown in section 3 , it follows from factorization of Wilson loop averages and $N$ counting. In section 4 , we will use our formalism to obtain a nontrivial new result for the potential energy between heavy quarks in the presence of an adjoint scalar field. For the massless case it is:

$$
V(L)=\sigma_{e f f} L-\frac{\pi}{24} \frac{1}{L}
$$

where $L$ is the distance between the quarks, and $\sigma_{\text {eff }}$ the (non-calculable) effective string tension, which is renormalized by the presense of adjoint loops. We cannot give a strict mathematical derivation of this formula, but it follows from some rather reasonable assumptions. We want to stress that we do not know of any way to derive this relation in the usual second quantized formulation of the theory.

Before obtaining these results, we derive, in section 2, the "first quantized" version of the theory, starting from the more familiar second quantized one. We also give some results for expectation values of Wilson loops that will be used subsequently. For completeness we give some details of how to derive these results in an appendix. In section 5, we extend the results to the case of massive adjoint field and finally we summarize our results and discuss possible extensions in section 6 . 


\section{2. "First quantized" (loop) formulation of $\mathrm{AdQCD}_{2}$}

We start from the partition function of 1+1-dimensional QCD coupled to an adjoint scalar Higgs, $\Phi$, given by

$$
Z=\int \mathcal{D} A \mathcal{D} \Phi e^{-i S}
$$

where

$$
S=\int d^{2} x \operatorname{Tr}\left[\frac{1}{4} F_{i j} F^{i j}+\frac{1}{2}\left(\partial_{i} \Phi-g\left[A_{i}, \Phi\right]\right)^{2}+\frac{1}{2} m^{2} \Phi^{2}\right]
$$

and $A^{\mu}$ and $\Phi$ are in the adjoint representation of $S U(N), m$ is the mass of the Higgs field. We have chosen to study the theory without any scalar self-interaction. The $m$ appearing in the Lagrangian (3) is a bare mass that cannot directly be identified with the mass of the adjoint particles. We shall return to the question of mass renormalization in section 4 . We shall be particularly interested in the case when the renormalized mass vanishes, or at least satisfies $m \ll g$. It would be interesting to study this limit both in perturbation theory and on the lattice.

Note that in the dimensional reduction of our theory from a $3 \mathrm{~d}$ theory a mass term, a $\Phi^{4}$ term and higher order potential terms $\sim \Phi^{2 n}$ occur. The coefficients, which are functions of $\mathrm{T}$, are calculable in perturbation theory if proper care is taken to deal with the infrared divergences due to the vanishing mass term.

The expectation value of an external Wilson loop $W_{F}$ is given by

$$
\left\langle W_{F}\right\rangle_{\mathrm{QCD}}=\frac{1}{Z} \int \mathcal{D} A \mathcal{D} \Phi e^{i S} W_{F}
$$

where $S$ is defined by (3).

The next step is to convert the integration over $\Phi$ in (2) to an integration over loops. This is achieved by firstly integrating out the field $\Phi$ and then reexpressing the propagator in terms of particle variables as a Feynman path-integral [21, 4]. 
For the non-Abelian case with scalar particles we obtain

$$
\begin{gathered}
\int \mathcal{D} \Phi e^{-\frac{i}{2} \int d^{2} x \operatorname{Tr}\left[\left(\partial_{i} \Phi-g\left[A_{i}, \Phi\right]\right)^{2}+\frac{1}{2} m^{2} \Phi^{2}\right]}=\sum_{n=0}^{\infty} \frac{1}{n !} \\
\prod_{l=1}^{n} \int_{0}^{\infty} \frac{d T_{l}}{T_{l}} \int_{x_{l}(0)=x_{l}(T)} D x_{l} e^{i \sum_{l=1}^{n} \oint_{0}^{T_{l}} d \tau_{l}\left[\frac{1}{2} m^{2}+\frac{1}{2} \dot{x}_{l}^{2}\left(\tau_{l}\right)\right]} \operatorname{Tr}_{A}\left(P e^{-i \oint d \tau_{l} A_{i}\left(x_{l}\right) \dot{x}_{l}^{i}\left(\tau_{l}\right)}\right)
\end{gathered}
$$

where $\tau_{l}$ parametrizes the path, the dot stands for derivative with respect to $\tau_{l}$, the subscript $A$ means that the trace is taken in the adjoint representation and $P$ denotes path-ordering. In obtaining the last expression a regulator has been assumed and an infinite constant has been dropped.

Inserting (5) into (2) and rearranging the terms yields

$$
Z=\sum_{n=0}^{\infty} \frac{1}{n !} \prod_{l=1}^{n} \int_{0}^{\infty} \frac{d T_{l}}{T_{l}} \oint D x_{l} e^{i \sum_{l=1}^{n} \oint_{0}^{T_{l}} d \tau_{l}\left[\frac{1}{2} m^{2}+\frac{1}{2} \dot{x}_{l}^{2}\left(\tau_{l}\right)\right]}\left\langle\prod_{l=1}^{n} W_{A_{l}}\right\rangle
$$

where the last expectation value of Wilson loops is with respect to the pure YangMills action, and the normalization is such that $\langle 1\rangle=1$.

Finally, using (4) and (6), we can derive the following expression for the expectation value $\left\langle W_{F}\right\rangle$ of a Wilson loop:

$$
\left\langle W_{F}\right\rangle_{\mathrm{QCD}}=\sum_{n=0}^{\infty} \frac{1}{n !} \prod_{l=1}^{n} \int_{0}^{\infty} \frac{d T_{l}}{T_{l}} \oint D x_{l} e^{i \sum_{l=1}^{n} \oint_{0}^{T_{l}} d \tau_{l}\left[\frac{1}{2} m^{2}+\frac{1}{2} \dot{x}_{l}^{2}\left(\tau_{l}\right)\right]}\left\langle W_{F} \prod_{l=1}^{n} W_{A_{l}}\right\rangle^{c}
$$

where the connected part of the expectation value of products of Wilson loops is defined as connected with respect to the external loop, i.e.

$$
\begin{aligned}
\left\langle W_{F} W_{A}\right\rangle^{c} & =\left\langle W_{F} W_{A}\right\rangle-\left\langle W_{F}\right\rangle\left\langle W_{A}\right\rangle \\
\left\langle W_{F} W_{A_{1}} W_{A_{2}}\right\rangle^{c} & =\left\langle W_{F} W_{A_{1}} W_{A_{2}}\right\rangle-\left\langle W_{F} W_{A_{1}}\right\rangle^{c}\left\langle W_{A_{2}}\right\rangle-\left\langle W_{F} W_{A_{2}}\right\rangle^{c}\left\langle W_{A_{1}}\right\rangle \\
& -\left\langle W_{F}\right\rangle\left\langle W_{A_{1}} W_{A_{2}}\right\rangle
\end{aligned}
$$

etc.

The fact that the expectation value (7) depends only on the connected loops can be 
shown by standard arguments. Similarly the partition function can be expressed as,

$$
Z=e^{\langle W\rangle+\frac{1}{2}\langle W W\rangle^{c}+\frac{1}{3 !}\langle W W W\rangle^{c}+\ldots}
$$

where the superscript $c$ here stands for the fully connected averages.

$$
\begin{aligned}
\langle W W\rangle^{c} & \equiv\langle W W\rangle-\langle W\rangle\langle W\rangle, \\
\langle W W W\rangle^{c} & \equiv\langle W W W\rangle-3\langle W\rangle\langle W W\rangle+2\langle W\rangle\langle W\rangle\langle W\rangle, \text { etc. }
\end{aligned}
$$

The formula (9) is essentially an expansion in terms of cummulants.

\section{Comments:}

The relations (6) and (7) define a massive $\mathrm{AdQCD}_{2}$ in terms of classical trajectories of particles of mass $m$ and Wilson loop averages in the adjoint representation.

The expression for $\left\langle W_{F}\right\rangle_{\mathrm{QCD}}$ is in terms of connected green functions, just as an S-matrix element in ordinary field theory.

For the case of spinning particles the integrand in the right hand side of (5) must

be multiplied by a spin factor. In two dimensions this is simply $e^{i \pi(\nu+1)}$, where $\nu$ is the number of self-intersections of the loop, as originally obtained by Strominger [4]. For a general discussion of spin factors in higher dimensions, see e.g.[22].

\section{Expectation values of Wilson loops and the $N \rightarrow \infty$ limit}

As mentioned in the introduction, we will try to gain a partial understanding of the theory defined by (6). For this purpose, we shall now discuss expectation values of Wilson loops of the type occuring in (6) and (7). These can be obtained using methods given in $[19,6,12,13,20]$ and we shall here only state the results. For completeness, some of the techniques and sample calculations are summarized in the appendix.

We first consider the configuration of $n$ disconnected adjoint loops inside the external fundamental one, see fig. 1a. We can assume the space outside of the external loop to have arbitrary topology, but we take its area to be infinite. Following 
the method discussed in appendix we derive the expression:

$$
\begin{aligned}
& \left\langle W_{F} W_{\left.A_{1} \ldots W_{A_{n}}\right\rangle=}\right. \\
& \frac{e^{-\frac{g_{0}^{2}}{N}\left(S_{F}-\sum_{i=1}^{n} S_{i}\right) C_{F}}}{\left(d_{F}\right)^{n-1}} \prod_{i=1}^{n}\left(d_{F} e^{-\frac{g_{0}^{2}}{N} S_{i} C_{F}}+d_{R_{1}} e^{-\frac{g_{0}^{2}}{N} S_{i} C_{R_{1}}}+d_{R_{2}} e^{-\frac{g_{0}^{2}}{N} S_{i} C_{R_{2}}}\right)
\end{aligned}
$$

where $S_{i}$ is the area inside the $i^{\text {th }}$ adjoint loop and $S_{F}$ the area of the fundamental loop. By $C_{R}$ we denote the quadratic casimir of the representation $R$ and $R_{1}, R_{2}$ are defined by the decomposition $F \otimes A=F \oplus R_{1} \oplus R_{2}$. We have also redefined the QCD coupling constant $g^{2}$ by $\frac{g_{0}^{2}}{N}$, so that in the large $N$ limit $g_{0}^{2}$ remains finite.

Before we proceed to present more results of a similar kind, it is instructive to give a string interpretation of the relation (11). Remember that the expectation value of a Wilson loop in the fundamental representation with respect to pure Yang-Mill's action is $\left\langle W_{F}\right\rangle=d_{F} e^{-\frac{g_{0}^{2}}{N} S_{F} C_{F}}$ so the tension of a fundamental string is, $\sigma_{F}=\frac{g_{0}^{2}}{N} C_{F}$, which in the large $N$ limit equals $\frac{g_{0}^{2}}{2}$. Similarly the exponents of the three terms inside the parenthesis in (11) can be associated with the three different values for the string tension corresponding to the three different representations in the product $F \otimes A$. The corresponding prefactors $d_{R}$ give the probability for the different tensions. In the large $N$ limit where an adjoint particle can be thought simply of as a $\bar{F} F$ combination, we have the string configurations $\bar{F}-A-A-F$ and $\bar{F}-A \equiv A-F$, corresponding to $\sigma_{F}$ and $\sigma_{R_{1}}=\sigma_{R_{2}}=3 \sigma_{F}$, respectively.

We also give the result for the configuration with two non-intersecting adjoint loops, one inside the other, see fig. 1b. It is given by

$$
\begin{gathered}
\left\langle W_{F} W_{A} W_{A}\right\rangle= \\
e^{-\frac{g_{0}^{2}}{N}\left(S_{F}-S_{2}\right) C_{F}}\left(e^{-\frac{g_{0}^{2}}{N}\left(S_{2}-S_{1}\right) C_{F}}\left[d_{R_{1}} e^{-\frac{g_{0}^{2}}{N} S_{1} C_{R_{1}}}+d_{R_{2}} e^{-\frac{g_{0}^{2}}{N} S_{1} C_{R_{2}}}+d_{F} e^{-\frac{g_{0}^{2}}{N} S_{1} C_{F}}\right]\right. \\
\left.+e^{-\frac{g_{0}^{2}}{N}\left(S_{2}-S_{1}\right) C_{R_{1}}} \sum_{K_{i}} d_{K_{i}} e^{-\frac{g_{0}^{2}}{N} S_{1} C_{K_{i}}}+e^{-\frac{g_{0}^{2}}{N}\left(S_{2}-S_{1}\right) C_{R_{2}}} \sum_{L_{j}} d_{L_{j}} e^{-\frac{g_{0}^{2}}{N} S_{1} C_{L_{j}}}\right)
\end{gathered}
$$

where $S_{1}$ and $S_{2}$ are the areas of the adjoint loops (with $S_{2}>S_{1}$ ), $S_{F}$ is the area of the fundamental, $K_{i}$ and $L_{j}$ are the irreducible components obtained from the 
decompositions of $R_{1} \otimes A$ and $R_{2} \otimes A$ respectively. The generalization of this to $\mathrm{n}$ adjoint loops with one inside the other is straightforward, but complicated to write in a closed form. We see that as one adds more and more adjoint loops, one inside the other, the string tension receives contributions from higher and higher representations. All contributions are multiplied by an entropy factor, which is simply the dimension of the representation.

Expectation values for (self)intersecting loops are harder to calculate, and the methods are briefly discussed in the appendix. We again record some results. For the configuration of two intersecting adjoint loops of areas $S_{1}+S$ and $S_{2}+S$, see fig. 2a, we get $\left\langle W_{A} W_{A}\right\rangle=e^{-\frac{g_{0}^{2}}{N} S_{1} C_{A}} e^{-\frac{g_{0}^{2}}{N} S_{2} C_{A}} \sum_{i} d_{A_{i}} e^{-\frac{g_{0}^{2}}{N} S C_{A_{i}}}$, where $A_{i}$ are defined by $A \otimes A=\sum_{i} A_{i}$. For a self-intersecting loop of total area $S_{1}+S_{2}$ and one point of intersection, as shown in fig. $2 \mathrm{~b}$, it is $\left\langle W_{A}\right\rangle=d_{A} e^{-\frac{g_{0}^{2}}{N}\left(S_{1}+S_{2}\right) C_{A}}$. This last result can be trivially generalized to a loop with $n$ self-intersectings but no overlapping areas. In this case the area in the exponent of the last expression is replaced by the total area $\left(\sum_{i=1}^{i=n} S_{i}\right)$. In fig. 2 c, finally we show a self-intersecting loop with overlapping areas. It is $\left\langle W_{A}\right\rangle=e^{-\frac{g_{0}^{2}}{N} S_{1} C_{A}} \sum_{R_{i}} d_{R_{i}} \alpha_{i} e^{-\frac{g_{0}^{2}}{N} S_{2} C_{R_{i}}}$, where $R_{i}$ and $\alpha_{i}$ are defined by the decomposition $\operatorname{Tr}_{A}\left(U^{2}\right)=\sum_{R_{i}} \operatorname{Tr}_{R_{i}}(U) \alpha_{i}$.

For large $N$, expectation values of products of Wilson loops factorize. This follows from general arguments and can easily be shown in our formulation, using some simple group theoretical rules given in the appendix. For instance, the relations (11) and (12) become

$$
\begin{aligned}
\left\langle W_{F} W_{A} \ldots W_{A}\right\rangle & =d_{F} e^{-\frac{g_{0}^{2}}{N} S_{F} C_{F}} \prod_{i} d_{A} e^{-\frac{g_{0}^{2}}{N} S_{i} C_{A}}[1+O(1 / N)] \\
\left\langle W_{F} W_{A} W_{A}\right\rangle & =d_{F} e^{-\frac{g_{0}^{2}}{N} S_{F} C_{F}} d_{A} e^{-\frac{g_{0}^{2}}{N} S_{2} C_{A}} d_{A} e^{-\frac{g_{0}^{2}}{N} S_{1} C_{A}}[1+O(1 / N)]
\end{aligned}
$$

Due to this factorization, the leading term in $N$ will cancel in the relations (8) . The sub-leading contributions can however not be ignored in the calculation of $\left\langle W_{F}\right\rangle_{Q C D}$, since they are of the same order as $\left\langle W_{F}\right\rangle$. To see this, notice that in $\left\langle W_{F} W_{A}\right\rangle^{c}$ it is the sub-leading part of $\left\langle W_{F} W_{A}\right\rangle$ which contributes, in $\left\langle W_{F} W_{A} W_{A}\right\rangle^{c}$ 
it is the sub-sub-leading term of $\left\langle W_{F} W_{A} W_{A}\right\rangle$ which contributes and so on. The sub-leading part of $\left\langle W_{F} W_{A}\right\rangle$, the sub-sub-leading part of $\left\langle W_{F} W_{A} W_{A}\right\rangle$ and so on are $O(N)$, thus all these connected expectation values are $O(N)$. Expressions similar to (7) and (13) can easily be found for the 't Hooft model, i.e. for insertion of fundamental loops. The difference in this case is that the sub-leading term is $O(1)$, and can thus be neglected in the large $N$ limit in the calculation of the expectation value $\left\langle W_{F}\right\rangle_{Q C D}$. We have therefore obtained, in the loop formulation, the well known result that the fundamental loops do not renormalize the string tension, while the adjoint ones do. Similarly, the sub-leading parts of the connected averages in (9) can be neglected for the case of fundamental loops but cannot be neglected when the loops are in the adjoint representation. This, again, is a manifestation of the 't Hooft planar rule in the loop formulation.

\section{Large loop contributions to $W_{F}$}

In this section we shall estimate the contribution from a certain class of large (compared to $g^{-2}$ ) adjoint loops to the expectation value of a large fundamental loop, and thus to the static potential between heavy "quarks". We start with the following observations:

i) In the expectation value $\left\langle W_{F} W_{A_{1} \ldots . .} W_{A_{n}}\right\rangle$, where all the adjoint loops are contained within the fundamental loop, there is always one term $\sim e^{-g^{2} S_{F} C_{F}}$ since $F$ occurs once in the decomposition $F \otimes A$. In the string language, this corresponds to the possibility of putting an adjoint particle somewhere in a fundamental string without changing its tension on either side.

ii) For expectation values $\left\langle W_{F} W_{A_{1}}^{L} \ldots W_{A_{n}}^{L}\right\rangle$ where all adjoint loops are large, i.e. with an area $\gg g^{-2}$, the above term will be exponentially large compared with all other contributions. A simple example is shown in fig. 1a.

iii) Configurations with at least one loop extending outside the fundamental loop are exponentially suppressed as illustrated in fig. 3 . 
Even though true for the configurations in fig. $1 \mathrm{a}$ and $2 \mathrm{~b}$, the statements ii) and iii) are not strictly true. We will return to this question below.

The basic idea in this paper is as follows:

Consider a configuration with only large loops, $W_{A}^{L}$, that are all confined within the fundamental one. For this case we can, with exponential accuracy, make the replacement

$$
\left\langle W_{F} W_{A_{1}}^{L} \ldots . W_{A_{n}}^{L}\right\rangle \rightarrow e^{-g^{2} C_{F} S_{F}}
$$

at least in the generic case where the areas of intersection are also large. In the presence of small loops that do not intersect the large ones, we similarly have

$$
\left\langle W_{F} W_{A_{1}}^{S} \ldots . W_{A_{m}}^{S} W_{A_{1}}^{L} \ldots . W_{A_{n}}^{L}\right\rangle \rightarrow\left\langle W_{F} W_{A_{1}}^{S} \ldots . W_{A_{m}}^{S}\right\rangle
$$

Let us for the moment neglect the small loops that intersect the large ones and try to understand the meaning of (14) and (15). That we can remove the large loops from the gauge field averages means that they are non-interacting. The string version of this statement is that an adjoint particle can be put on the string of fundamentals in such a way that it feels no net force, since the string tension is the same on both its sides. This does not mean, however, that the large loops correspond to those of a completely free field theory since they have to be "confined" withing the contour of the fundamental loop in order not to be suppressed. Below we shall argue that the sum of these non-interacting, but "confined loops", is simply related to the Casimir energy of a free field theory.

Let us now return to the small loops intersecting one or several of the large ones. Those intersecting a single large loop will generically give a contribution that is proportional to the length of the large loop. It is natural to identify this as a mass renormalization of the particles in the large loops. Similarly the small loops intersecting two large loops will give rise to a short range interaction between them. Neither of these statements can be proven, but they are reasonable assumptions. In the following we will be interested in the long wavelength properties of the 
theory, and therefore keep the mass renormalization but neglect any short range interaction. We will comment on this approximation in the last section.

In order to express these assumptions formally, we separate the integration over $x_{l}$ in the exponent of the expression (7) into large and small loop contribution. Then, according to the discussion above, the Wilson loops $W_{A_{i}}^{L}$ for the large loops can be removed from the expectation value in the presence of the external fundamental loop. (Notice that with an exponential accuracy the large Wilson loops can be also removed from the connected expectation values.) Thus (7) can be written as

$$
\left\langle W_{F}\right\rangle \simeq e^{\int_{0}^{\infty} \frac{d T}{T} \int_{\text {Large }} D x e^{i S_{r}}}\left\langle\exp \left(\int_{0}^{\infty} \frac{d T}{T} \int_{\text {Small }} D x e^{i S_{b}} W_{A}\right) W_{F}\right\rangle^{c}
$$

where the subindices $r$ and $b$ in the definition of $S_{r}$ and $S_{b}$ refer to the renormalized and bare mass respectively; that is, $S_{r}=\int_{0}^{T} d \tau\left(\frac{1}{2} m_{r}^{2}+\frac{1}{2} \dot{x}^{2}\right)$ and $S_{b}=\int_{0}^{T} d \tau\left(\frac{1}{2} m_{b}^{2}+\right.$ $\frac{1}{2} \dot{x}^{2}$ ). Let us emphasize again that the integral in (16) is taken over loops contained inside the fundamental loop.

We now make the following assumptions:

i) The large loops that are "confined" within the contour of the fundamental loop can effectively be taken into account by imposing appropriate boundary conditions. It will be important that the quantities we calculate do not depend on the details of these conditions, as will be discussed below.

ii) The (non-calculable) last factor in (16) will effectively only renormalize the string tension. This is almost trivially true, since the tension is the only quantity that characterizes the low-energy behaviour of a one-dimensional string. (In higher dimensions there could be terms related to extrinsic curvature [23], and for highly excited states one will of course "see" the adjoint degrees of freedom.)

Since the expectation value in (16) that depends on the small loops factorizes, and has the form of the Wilson loop average (7), assumption ii) implies that it 
will contribute a factor $e^{-\sigma(R) S_{F}}$ to $\left\langle W_{F}\right\rangle$. Here the string tension $\sigma(R)$ depends on the cutoff $R$ which defines the meaning of large and small. From the previous discussion it should be clear that we must regulate in such a way as $L>>R>>\frac{1}{g}$.

The other factor in (16), that depends on the large loops, has the form of a partition function of a confined scalar field theory, and is thus directly connected to the vacuum energy which, as we shall argue below, is of the form

$$
E_{v a c ; 2}=\frac{\lambda}{R^{2}} L-\frac{\pi}{24} \frac{1}{L}
$$

where $\lambda$ is a constant and the subindex 2 stands for the dimension of space-time. Thus, assuming i) and ii), we can write an explicit formula for a rectangular Wilson loop with area $S=L T$ in the limit $T \gg L \gg g^{-1}$ :

$$
\left\langle W_{F}\right\rangle=e^{-E_{t o t} T}
$$

where

$$
E_{t o t}=\sigma_{e f f} L+2 \delta M-\frac{\pi}{24} \frac{1}{L}
$$

is the total energy of a color singlet pair of static quarks. The last term in (19) is the Casimir energy of a free, massless field confined to the line-segment $L$ and $\delta M$ is a non-calculable mass renormalization of the heavy external source. This will arise from small loops intersecting the external in analogy with the mass renormalization of the large adjoint loops. Note that the term $\sim L / R^{2}$ in (17) must cancel against an identical piece in the cutoff dependent term $\sigma(R) \mathrm{L}$ coming from the small loops, in order to give a cutoff independent effective string tension $\sigma_{e f f}$. Finally, notice also that the approximation of the large loops being free holds only for finite $N$. All the results in this section are thus true only for finite $N$.

We now explain how we arrived at (17). The vacuum energy of a free field theory defined in a spatial volume $V$ is ultraviolet divergent. In addition to the usual free space divergence $\sim V \Lambda^{d}$, where $\Lambda$ is an ultraviolet cutoff and $d$ the 
space-time dimension, there are less singular terms depending on the particular boundary. In our case, i.e. for $d=2$, the expansion is

$$
E_{v a c ; 2}=L \Lambda^{2}+\frac{c}{L}
$$

A convenient way to exhibit the divergence structure is by using the so called multiple reflection expansion (MRE) method. The vacuum energy is related to the Euclidean propagator $G\left(x, x^{\prime} ; i \beta\right)$, with $\beta$ denoting the Euclidean time, via,

$$
E_{v a c}=\lim _{\beta \rightarrow 0} \frac{1}{2} \int d x_{x^{\prime} \rightarrow x}\left(-\partial_{\beta}^{2}+\nabla_{x}^{2}\right) G\left(x, x^{\prime} ; i \beta\right)
$$

where $G\left(x, x^{\prime} ; i \beta\right)$ is defined with the appropriate boundary conditions at the end points of the segment. In the MRE-method one expands this propagator in a series in $\mathrm{n}$, where the $n^{\text {th }}$ term is the configuration with $\mathrm{n}$ reflections in the boundaries and free propagation between the reflections. For a scalar field in $1+1$ dimension, the propagator is given by

$$
G^{N}\left(x, x^{\prime} ; i \beta\right)=\sum_{n=-\infty}^{n=\infty}( \pm 1)^{n} G^{0}\left(x, x_{n}^{\prime} ; i \beta\right)
$$

where $x_{n}^{\prime}=(-1)^{n} x^{\prime}+n L$, with $\left|x^{\prime}\right|<\frac{L}{2}$, is the position of the $n$th mirror image of the point $x^{\prime}$ and $L$ is the volume. The + and - signs refer to Neuman and Dirichlet boundary conditions respectively. It is then straightforward to show that the leading divergence in (20) comes from the $n=0$ term in (22) and the Casimir energy from the terms with $n$ even. (The terms in the expansion with $n$ odd give zero.) Summing the contributions with at least two reflections, we get

$$
E_{v a c}=\frac{2}{\pi \beta^{2}} L-\frac{\pi}{24} \frac{1}{L}
$$

irrespectively of whether the boundary conditions are Dirichlet or Neuman. The origin of the last term in (19) should now be clear. It is the Casimir energy. It is an 
assumption that the "confinement" of the loops, which in the QCD case is due to the exponential suppression of loops extending beyond $W_{F}$, can be replaced by a simple, "confining" boundary condition. In the framework of the MRE this is very natural since we can think of the exponential supression as providing a reflection of the particle, and summing over all loops will, as usual, reproduce the quantum mechanical propagator. In this context it is pleasing that, in the two cases we have considered, the result does not depend on the specific boundary condition. For practical calculations one uses either a momentum cutoff or a point splitting procedure. However, since the divergence structure cannot depend on the details of the cutoff, a cutoff in the length of the loops should be equally good, so we can replace $\Lambda$ with $1 / R$. Notice that our result for the Casimir energy is consistent with the finite scaling results of quantum field theory [24]. In fact, every conformal field theory in $1+1$ dimensions with central charge equal to 1 contributes a factor

equal to $-\frac{\pi}{24 L}$ as the finite-size corrections to the free-energy of an infinite long strip of width $L$, when the boundary conditions are appropriately chosen.

\section{The massive case}

So far we have considered only the $m_{r}=0$ case. The above analysis, however, can be extended for the case where $m_{r} \neq 0$. As we shall see, the most important effect, i.e. the $L$-dependent part in the Casimir energy in (19), will be negligible in the limit where $L>>m_{r}^{-1}$. The vacuum energy for the case of massive scalar field is given by

$$
E_{v a c}=\sum_{n} \frac{1}{2} \omega_{n}=\lim _{\beta \rightarrow 0}\left(-\frac{\partial Z}{\partial \beta}\right)
$$

where $Z=\sum_{n} e^{-\beta \frac{\omega_{n}}{2}}$ and $\omega_{n}=\sqrt{k_{n}^{2}+m^{2}}$, with $k_{n}=\frac{\pi n}{L}, n=1,2, \ldots$

From (24) we obtain

$$
\begin{aligned}
E_{v a c} & =\frac{2}{\pi \beta^{2}} L-\frac{m^{2}}{4 \pi} \ln \left(\frac{m \beta}{4}\right) L-\frac{3 m^{2}}{8 \pi} L+\frac{\gamma m^{2}}{4 \pi} L+\frac{\pi}{48 L}\left(1+\frac{4 m^{2} L^{2}}{\pi^{2}}\right)^{-\frac{1}{2}} \\
& -\frac{\pi}{16 L} \sqrt{1+\frac{4 m^{2} L^{2}}{\pi^{2}}}-\frac{m^{2} L}{4 \pi} \ln \left[\frac{\pi}{2 m L}\left(1+\sqrt{1+\frac{4 m^{2} L^{2}}{\pi^{2}}}\right)\right]
\end{aligned}
$$


To get some feeling for this result we shall consider different limis. First, when $m L$ goes to zero, with $L$ fixed, we get

$$
E_{v a c} \rightarrow \frac{2}{\pi \beta^{2}} L-\frac{m^{2}}{\pi} \ln \left(\frac{\beta m}{2}\right) L+\left(-\frac{5 m^{2}}{3 \pi}+\frac{\gamma m^{2}}{\pi^{2}}+\frac{m^{2}}{4 \pi} \ln \left(\frac{m L}{\pi}\right)\right) L-\frac{\pi}{24} \frac{1}{L}
$$

In the limit $m \rightarrow 0$ this gives the massless result (23), as expected. Notice that the terms proportional to $L$ in (26) contribute to the string tension renormalization, while the last term can be interpreted as the Casimir energy of a massive field.

In the limit where $m L>>1$ the expression (25) becomes

$$
E_{v a c} \rightarrow \frac{2 L}{\pi \beta^{2}}-\left(\frac{1}{4 \pi} \ln \left(\frac{m \beta}{4}\right)+\frac{3}{8 \pi}+\frac{\gamma}{4 \pi}\right) m^{2} L-\frac{m}{4}
$$

In order for the last limit to have a meaning, however, we must have $m<<\frac{1}{\beta}$, since here $\beta$ plays the role of $R$, the cutoff for the size of the loops, and $L \leq \frac{1}{m}$, which is the Compton wave length.

As in the massless case we take for granted that the cutoff dependent pieces in (25) combines with the non-calculable contributions from the small loops to give a cutoff independent effective string tension. Thus we have a prediction for the Wilson loop average for any value of the renormalized mass.

We conclude this section with a calculation of the effective string tension in the limit where $m>>g$. For this purpose we expand the effective action of the gauge field, $\Gamma[A]$ in terms of $\frac{g^{2}}{m^{2}}$. The leading contribution comes from the one-loop, vacuum polarization tensor, which is $\sim \frac{g^{2}}{m^{2}}$; namely, $\Pi^{\mu \nu}(k)=\left(g^{\mu \nu} k^{2}-k^{\mu} k^{\nu}\right) \Pi\left(k^{2}\right)$. In this case, the expansion gives

$$
\Pi\left(k^{2}\right)=-\frac{g^{2}}{m^{2}} \frac{1}{12 \pi}\left(g^{\mu \nu} k^{2}-k^{\mu} k^{\nu}\right)+\frac{g^{2}}{2 \pi} g^{\mu \nu}\left(\ln \frac{4 \pi \mu^{2}}{m^{2}}+\Psi(1)\right)
$$

\footnotetext{
* This calculation was suggested to us by Ismail Zahed.
} 
Therefore the renormalized propagator is given by

$$
\frac{g^{2}}{k^{2}} \frac{1}{1+\Pi\left(k^{2}\right)} \simeq \frac{g_{e f f}^{2}}{k^{2}}
$$

where

$$
g_{e f f}^{2}=g^{2}\left(1+\frac{C_{A}}{12 \pi} \frac{g^{2}}{m^{2}}\right)
$$

and $C_{A}$ is the second Casimir of the adjoint representation. Since the correct Coulomb energy is $E_{C} \sim g_{\text {eff }}^{2} L$, it is clear, that the expression (30) gives a correction to the string tension. Note that the effective string tension increases due to the presence of the heavy scalar.

\section{Discussion and outlook}

It should be clear from the previous two sections that our results for the heavy quark potential rests on heuristic arguments, and we want to comment upon their character.

First, we assumed results derived for specific configurations of Wilson loops to hold even in cases where we have not done explicit calculations. In particular, we assumed that curled up and intersecting loops will not qualitatively change the picture derived from configurations of smooth and non-intersecting ones. Such an assumption could well be dangerous, since we have no way of controlling the number of configurations of different types. Put differently, our arguments are all based on "energy" while we have no possibility to even estimate "entropy" effects. At least in a continuum theory, we know of no loop space techniques that would allow us to attack this problem - not even in principle.

Second, we made certain assumptions about the effective large-distance theory

of "large" loops. Some of these, like the presence of an effective string-tension and a mass renormalization of the large adjoint loops, we think that are rather safe. Our way to treat a sum of large confined and non-interacting loops by calculating 
vacuum energies á la Casimir is supported by the multiple reflection type arguments given in the text. However, the assumption that the large loops are non-interacting, on which this calculation rests, can certainly be doubted. It would be interesting to check, using perturbation theory, if our results are stable against adding a weak short range interaction in the vacuum energy calculation. However, calculations with both boundaries and interactions are notoriously complicated and, even in two dimensions, it is a non-trivial task.

Having given all the warnings, we shall now stress some interesting aspects of our result.

The presence of $\mathrm{a} \sim L^{-1}$ type correction to the string tension certainly is very reminiscent of Lüschers' universal finite size correction term in string theory [25]. In this context the correction is due to the transverse oscillations of the string, but the calculation is again that of a Casimir energy. Comparing coefficients, one finds that the $1 / L$ term we found in $(23)$ corresponds to a Lüscher term in $2+1$ dimensions. It is hard to believe that this is a coincidence given that the $\mathrm{AdQCD}_{2}$ can be thought as the dimensionally reduced theory from the $2+1$ high-Temperature QCD. We thus have the rather intriguing result that the 2 dimensional string remembers its 3 dimensional origin via the presence of the adjoint scalar field.

We also again want to stress that we know of no direct field theoretical way to arrive at our results.

Finally, we emphasize that in spite of all approximations, we have a very definite prediction for the heavy quark potential as a function of the renormalized mass. A lattice Monte Carlo simulation of the theory should tell us if we are on the right track or not.

Acknowledgements: We would like to thank M. Douglas, I.R. Klebanov, J. Greensite, J. Minahan, H. Nielsen, H. Rubinstein and I. Zahed for discussions. We especially thank A. P. Polychronakos for many enlightening conversations and a critical reading of the manuscript. 


\section{Appendix. Expectation values of Wilson loops}

In this appendix we discuss the method used in section 2 for computing the Wilson loop averages and taking the large $N$ limit. We also give a sample calculation. For the non-intersecting loops it is illuminating to use the Hamiltonian formalism of ref. [12]. In this formalism one lets time run in one direction and the Wilson loops are equal-time loops that wrap around the spacial direction. The variables of the theory are the Wilson loops $(\mathrm{W})$ while the states are given by the characters $\chi_{R}$ of the group in the specific representation. The propagation of a state in the representation $R$ through an area $S$ is achieved by the operator $e^{-\frac{g_{0}^{2}}{N} S C_{R}}$, where $C_{R}$ stands for the quadratic casimir of the representation $R$.

We first consider the configuration of a single adjoint loop inside a fundamental. We denote the quantity to be computed by $\left\langle W_{F} W_{A}\right\rangle$, where the subindices refer to the representations of the loop, fundamental or adjoint correspondingly. Because of the infinite limit of the outside area, the only state which propagates from $t=-\infty$ until the point of the fundamental loop insertion is the trivial one (vacuum). Then

$$
\left\langle W_{F} W_{A}\right\rangle=\int D W \chi_{F} e^{-\frac{g_{0}^{2}}{N} S_{1} C_{F}} \chi_{A} G\left(t_{A}-t_{F}\right) \sum_{\tilde{R}_{j}} \chi_{\tilde{R}_{j}} d_{\tilde{R}_{j}}
$$

where $\chi_{F}$ and $\chi_{A}$ denote the insertions of the fundamental and adjoint loops correspondingly, $G\left(t_{A}-t_{F}\right)$ stands for the propagator from the point of the fundamental loop insertion $t_{F}$ until the point of the adjoint loop $t_{A}$ and $\sum \chi_{\tilde{R}_{j}}$ is a complete set of states inserted at the time $t=\infty$. The factor $d_{\tilde{R}_{j}}$ denotes the dimension of the representation $\tilde{R}_{j}$ and it is inserted for each representation which reaches the "north" pole.

If $F \otimes A=R_{1} \oplus R_{2} \oplus F$, then for the characters of the group holds

$$
\chi_{F} \cdot \chi_{A}=\chi_{F \otimes A}=\chi_{R_{1}}+\chi_{R_{2}}+\chi_{F}
$$

Inserting the last expression in (31) and using the orthogonality condition

$$
\int D W \chi_{R_{i}}(W) \chi_{\tilde{R}_{j}}(W)=\delta_{R_{i}, \tilde{R}_{j}}
$$


we obtain

$$
\left\langle W_{F} W_{A}\right\rangle=e^{-\frac{g_{0}^{2}}{N}\left(S_{F}-S_{1}\right) C_{F}}\left(d_{F} e^{-\frac{g_{0}^{2}}{N} S_{1} C_{F}}+d_{R_{1}} e^{-\frac{g_{0}^{2}}{N} S_{1} C_{R_{1}}}+d_{R_{2}} e^{-\frac{g_{0}^{2}}{N} S_{1} C_{R_{2}}}\right)
$$

where $S_{F}$ and $S_{1}$ are the areas of the fundamental and adjoint loops respectively.

The Hamiltonian approach used in obtaining the last result is not suitable for intersecting or self-intersecting loops. The reason is that at the point of intersection the breaking of the space results to correlations between the two pieces and as a result the simple Hamiltonian rules are not applicable. Alternatively, one could use path-integral formulation in order to obtain the results for these more complicated topologies. Some explicit expression for (self)intersecting loops, obtained by this method, are given in section 3 in the text. Notice at this point that all results for (self)intersecting loops are for adjoint loops without the presence of the external fundamental loop. (The calculation of expectation values of (self)intersecting Wilson loops inside an external loop is a very difficult problem and has not been solved explicitly.)

In order to study the large $N$ limit of our theory we use the following group theoretical arguments for the dimension and the quadratic casimir of the product of two representations: assume that $\tilde{R}_{i}$ are the irreducible components contained in the decomposition of the product of the representations $R_{1}$ and $R_{2}$, that is, $R_{1} \otimes R_{2}=\sum_{i} \tilde{R}_{i}$, where $i=1, \ldots \nu$. Then, in the large $N$ limit there exist some representations $\tilde{R}_{l}$, where $l=1, . . \mu$ and $\mu<\nu$, such that to leading order in $N$ the dimension of the product of the representations are given by suming the dimensions of these leading components, namely, $\sum_{l} d \tilde{R}_{l}=d R_{1} \cdot d R_{2} \equiv d\left(R_{1} \otimes R_{2}\right)$. Moreover, the casimirs of these leading representations are all equal to the sum of the casimirs of the original ones, again, to leading order in $N$. Therefore for a decomposition of two such representations the $\tilde{R}_{l}$ are the ones that will contribute in the large $N$ limit.

Using these group theoretical rules in (34) we obtain the following large $N$ 
expression

$$
\left\langle W_{F} W_{A}\right\rangle=d_{F} e^{-\frac{g_{0}^{2}}{N} S_{F} C_{F}} d_{A} e^{-\frac{g_{0}^{2}}{N} S_{1} C_{A}}
$$

\section{REFERENCES}

1. G. 't Hooft, Nucl. Phys. B72 (1974) 461; B75 (1974) 461.

2. C.B. Thorn, Phys. Lett. B99 (1981) 458.

3. I. Bars and A. Hansson, Phys. Rev. D 13 (1976) 1744; I. Bars, Phys. Rev. Lett. 36 (1976) 1521; I. Bars, Nucl. Phys. B111 (1976) 413.

4. A. Strominger, Phys. Lett. 101B (1981) 271.

5. Yu.M. Makeenko and A.A. Migdal, Phys. Lett. 88B (1979) 135; A.A. Migdal, Phys. Rev. 175 (1980) 126.

6. V.A. Kazakov and I.K. Kostov, Nucl. Phys. B176 (1980) 199.

7. V.A. Kazakov, Nucl. Phys. B179 (1981) 283.

8. J. Polchinski, Phys. Rev. Lett. 68 (1992) 1267.

9. D. Gross, Nucl. Phys. B400 (1993) 161.

10. J.Minahan, Phys. Rev. D 47 (1993) 3430.

11. D. Gross and W. Taylor, Nucl. Phys. B400 (1993) 181 and Nucl. Phys. B403 (1993) 395.

12. J. Minahan and A. P. Polychronakos, Phys. Lett. B312 (1993) 155 and Nucl. Phys. B422 (1994) 172.

13. M. Douglas, RU-93-13, NSF-ITP-93-39, Mar. 1993. hep-th/9303159.

14. J. Greensite, Nucl. Phys. B249 (1985) 263 and Phys. Rev. D 40 (1989) 4167.

15. T.H. Hansson and I. Zahed, Phys. Lett. B309 (1993) 385.

16. S. Dalley and I. R. Klebanov, Phys. Rev. D 47 (1993) 2517. 
17. G. Bhanot, K. Demeterfi and I.R. Klebanov, Phys. Rev. D 48 (1993) 4980 and Nucl. Phys. B418 (1994) 15.

18. D. Kutasov, Nucl. Phys. B414 (1994) 33.

19. A. Migdal, Zh. Eksp. Teor. Fiz. 69 (1975) 810; B. Rusakov, Mod. Phys. Lett. A5 (1990) 693.

20. M. Blau and G. Thomson, Int. Jour. Mod. Phys. A A7 (1992) 3781.

21. K. Bardakci and S. Samuel, Phys. Rev. D 18 (1978) 2849.

22. A. M. Polyakov, in Fields, strings and critical phenomena, Proc. Les Houches Summer School, Vol. IL 1988, ed. E. Brézin and J. Zinn-Justin (NorthHolland, Amsterdam, 1990) p.305; J. Grundberg, T.H. Hansson and A. Karlhede, Nucl. Phys. B347 (1990) 420.

23. A. M. Polyakov, Nucl. Phys. B268 (1986) 406.

24. H. W. J. Blöte, J. L. Cardy and M. P. Nightingale, Phys. Rev. Lett. 56 (1986) 742; I. Affleck, Phys. Rev. Lett. 56 (1986) 746.

25. M. Lüscher, Nucl. Phys. B180[FS2] (1981) 317. 


\section{Figure Captions}

Fig. 1a. Non-intersecting adjoint loops inside a fundamental.

Fig. 1b. Overlapping, non-intersecting adjoint loops inside a fundamental.

Fig. 2a. Intersecting adjoint loops.

Fig. 2b. A self-intersecting adjoint loop.

Fig. 2c. A self-intersecting adjoint loop with overlapping areas.

Fig. 3. A configuration with a section, of area $S$, of a large adjoint loop outside

of the fundamental, is weighted by the factor $e^{-S \sigma_{A}}$, and therefore suppressed for large $S$. 
This figure "fig1-1.png" is available in "png" format from: http://arxiv.org/ps/hep-th/9410235v1 
This figure "fig1-2.png" is available in "png" format from: http://arxiv.org/ps/hep-th/9410235v1 